Proceedings of the XXXVIII International School and Conference on the Physics of Semiconductors "Jaszowiec" 2009

\title{
Electrical and Magnetic Studies of the $\mathrm{Cd}_{x} \mathrm{Cr}_{y} \mathrm{~V}_{z} \mathrm{Se}_{4}$ Spinels
}

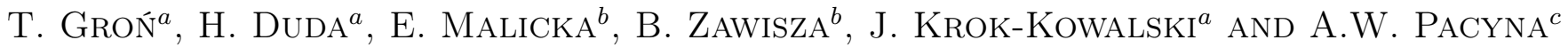 \\ ${ }^{a}$ Institute of Physics, University of Silesia, Uniwersytecka 4, 40-007 Katowice, Poland \\ ${ }^{b}$ Institute of Chemistry, University of Silesia, Szkolna 9, 40-006 Katowice, Poland
}

${ }^{c}$ The Henryk Niewodniczański Institute of Nuclear Physics, E. Radzikowskiego 152, 31-342 Kraków, Poland

The electrical and magnetic investigations carried out on the polycrystalline spinels with the general chemical formula $\mathrm{Cd}_{x} \mathrm{Cr}_{y} \mathrm{~V}_{z} \mathrm{Se}_{4}$ (where $z=0.06,0.12,0.24$, and 0.31 ) revealed semiconducting and ferromagnetic properties with a Curie temperature of $127.5 \mathrm{~K}$. A step-like structure of the electrical conductivity, $\sigma(T)$, was observed for the polycrystal containing $6 \% \mathrm{~V}$ ions in the octahedral sites while the remaining samples showed a typical Arrhenius behaviour of $\sigma(T)$. These effects are interpreted in terms of ferromagnetic spin clusters which finally dissolve on going towards the paramagnetic limit including non-stoichiometry.

PACS numbers: 72.80.Jc, 75.50.Pp, 75.50.Dd

\section{Introduction}

The magnetic spinel compounds reveal still the interesting phenomena. Recently, e.g. colossal magnetoresistance in $\mathrm{Cu}$ doped $\mathrm{FeCr}_{2} \mathrm{~S}_{4}$, gigantic Kerr rotation in $\mathrm{FeCr}_{2} \mathrm{~S}_{4}$, multiferroic behaviour and colossal magnetocapacitive effect in $\mathrm{CdCr}_{2} \mathrm{~S}_{4}$ and $\mathrm{HgCr}_{2} \mathrm{~S}_{4}$, large magnetostriction and negative thermal expansion in $\mathrm{ZnCr}_{2} \mathrm{Se}_{4}$ and a step-like structure of the electrical conductivity (SLS EC) in Ga doped $\mathrm{ZnCr}_{2} \mathrm{Se}_{4}$ and $\mathrm{CdCr}_{2} \mathrm{Se}_{4}$ were observed [1-5]. The SLS EC effect was first discovered in the $\mathrm{Zn}_{1-x} \mathrm{Ga}_{2 x / 3} \mathrm{Cr}_{2} \mathrm{Se}_{4}$ spinel system for the single crystal containing $3 \% \mathrm{Ga}$ ions in the octahedral sites [4]. It has been explained in terms of impurity conduction and hopping conduction due to ferromagnetic spin clusters which finally dissolve on going towards the paramagnetic limit. Similar behaviour of the electrical conductivity has been observed in the $\mathrm{CdCr}_{2-x} \mathrm{Ga}_{x} \mathrm{Se}_{4}$ spinel system for the single crystal containing $1.5 \% \mathrm{Ga}$ ions in the octahedral sites [5]. Monte Carlo simulations carried out on large samples of two-dimensional and three-dimensional Ising models confirmed that for critical concentrations (i.e., for the fraction of negative interactions and for the fraction of frustrated spins) the magnetic defect structures (clusters) appear leading to the unusual changes of the physical properties [6].

Pure $\mathrm{CdCr}_{2} \mathrm{Se}_{4}$ combines a $p$-type semiconducting behaviour of the Arrhenius type and ferromagnetic order with the Curie temperature $T_{\mathrm{C}}=142 \mathrm{~K}$ and the CurieWeiss temperature $\theta_{\mathrm{CW}}=190 \mathrm{~K}$ [7].

\section{Experimental details}

Powder samples of the $\mathrm{Cd}_{x} \mathrm{Cr}_{y} \mathrm{~V}_{z} \mathrm{Se}_{4}$ spinel series were obtained by a ceramic method [8]. The X-ray diffraction was done with the aid of the SIEMENS D5000 diffractometer. A precise atomic content determination of each sample with the aid of the energy-dispersive X-ray fluorescence spectrometry (EDXRFS) showed that the polycrystals of the $\mathrm{Cd}_{x} \mathrm{Cr}_{y} \mathrm{~V}_{z} \mathrm{Se}_{4}$ spinel system are non-stoichiometric, i.e. $x+y+z \neq 3$ (see Table I).
TABLE I

The chemical composition of the $\mathrm{Cd}_{x} \mathrm{Cr}_{y} \mathrm{~V}_{z} \mathrm{Se}_{4}$ spinels.

\begin{tabular}{c|c|c|c}
\hline \hline$x$ & $y$ & $z$ & $x+y+z$ \\
\hline 0.87 & 1.93 & 0.06 & 2.86 \\
0.87 & 2.07 & 0.12 & 3.06 \\
0.91 & 2.05 & 0.24 & 3.2 \\
0.88 & 1.96 & 0.31 & 3.15
\end{tabular}

The temperature dependences of mass susceptibility $\chi_{\sigma}(T)$, electrical conductivity $\sigma(T)$ and thermoelectric power $S$ were measured using: (1) a Cahn automatic magnetic electrobalance of the Faraday type, (2) a 4-point dc method, and (3) a differential method with the temperature gradient $\Delta T$ of about $5 \mathrm{~K}$, respectively. For electrical measurements the powder samples were compacted in disc form $(10 \mathrm{~mm}$ in diameter and $1-2 \mathrm{~mm}$ thick) using a pressure of $1.5 \mathrm{GPa}$ and they were next sintered during $2 \mathrm{~h}$ at $473 \mathrm{~K}$.

TABLE II

The electrical and magnetic parameters of the $\mathrm{Cd}_{x} \mathrm{Cr}_{y} \mathrm{~V}_{z} \mathrm{Se}_{4}$ spinels.

\begin{tabular}{c|c|c|c|c|c}
\hline \hline$z$ & $T_{\mathrm{C}}$ & $\theta_{\mathrm{CW}}$ & $\mu_{\mathrm{eff}}$ & \multicolumn{2}{|c}{$E_{\mathrm{A}}[\mathrm{eV}]$} \\
\cline { 5 - 6 } & {$[\mathrm{K}]$} & {$[\mathrm{K}]$} & {$\left[\mu_{\mathrm{B}} /\right.$ f.u. $]$} & $100 \mathrm{~K}$ & $273 \mathrm{~K}$ \\
\hline 0.06 & 128 & 160 & 5.83 & 0.05 & 0.27 \\
0.12 & 128 & 157 & 6.09 & 0.06 & 0.26 \\
0.24 & 127 & 147 & 5.3 & 0.06 & 0.24 \\
0.31 & 127 & 140 & 5.7 & 0.06 & 0.22
\end{tabular}

\section{Results and discussion}

The structural studies of the $\mathrm{Cd}_{x} \mathrm{Cr}_{y} \mathrm{~V}_{z} \mathrm{Se}_{4}$ spinels revealed a single-phase material with the cubic spinel structure of the $\mathrm{AB}_{2} \mathrm{X}_{4}$ type (where $\mathrm{A}=\mathrm{Cd}, \mathrm{B}=\mathrm{Cr}$ and $\mathrm{V}$, 
$\mathrm{X}=\mathrm{Se}$ ) and the space group $F d 3 m$ (No. 227) in the compositional range $0.06 \leq z \leq 0.31$ while the $\mathrm{CdV}_{2} \mathrm{Se}_{4}$ end member crystallizes in the monoclinic structure and its unit cell parameters refined by least squares method are as follows: $a=1077.22 \mathrm{pm}, b=696.35 \mathrm{pm}$, $c=1053.98 \mathrm{pm}, \beta=112.42^{\circ}$.

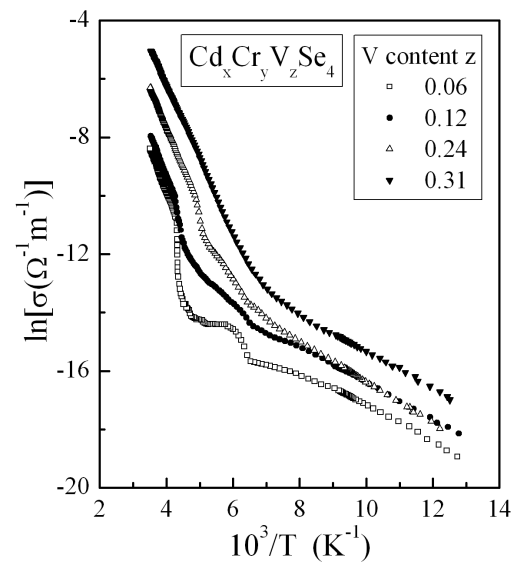

Fig. 1. The electrical conductivity $(\ln \sigma)$ vs. reciprocal temperature $T^{-1}$ for polycrystals with $z=0.06,0.12$, 0.24 , and 0.31 of the $\mathrm{Cd}_{x} \mathrm{Cr}_{y} \mathrm{~V}_{z} \mathrm{Se}_{4}$ spinel system.

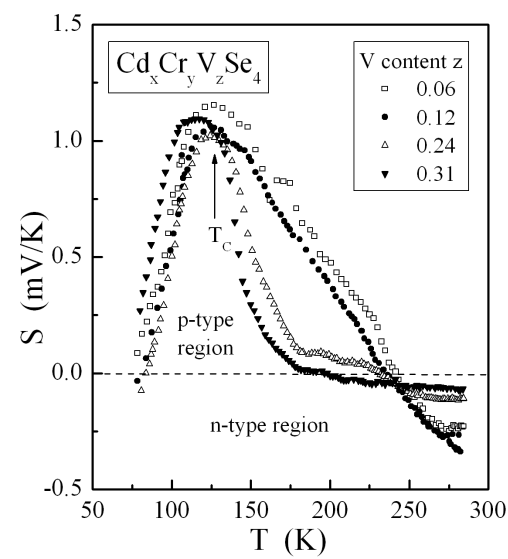

Fig. 2. The thermoelectric power $S$ vs. temperature $T$ for polycrystals with $z=0.06,0.12,0.24$, and 0.31 of the $\mathrm{Cd}_{x} \mathrm{Cr}_{y} \mathrm{~V}_{z} \mathrm{Se}_{4}$ spinel system. $T_{\mathrm{C}}$ is marked by arrow.

The electrical and magnetic measurements depicted in Figs. 1-3 and in Table II showed that the spinels under study are ferromagnets and thermally activated semiconductors. A transition from ferro- to paramagnetic state at the Curie temperature $T_{\mathrm{C}}$ well correlates both with a $p$-type thermopower maximum and a change of electrical conductivity from extrinsic to intrinsic for low and higher values of the energy activation $E_{\mathrm{A}}$, respectively, suggesting an electron-phonon coupling. With an increase of $\mathrm{V}$ content $z$ the Curie-Weiss temperature $\theta_{\mathrm{CW}}$ and $E_{\mathrm{A}}$ in the intrinsic region decrease while the effective magnetic moment $\mu_{\text {eff }}$ slowly fluctuates. For all samples the $n$-type

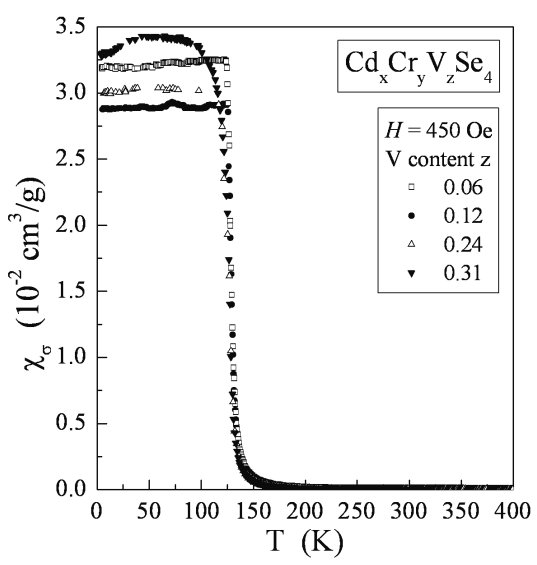

Fig. 3. The dc mass susceptibility $\chi_{\sigma}$ vs. temperature $T$ for polycrystals with $z=0.06,0.12,0.24$, and 0.31 of the $\mathrm{Cd}_{x} \mathrm{Cr}_{y} \mathrm{~V}_{z} \mathrm{Se}_{4}$ spinel system recorded at $H=450 \mathrm{Oe}$

electrical conductivity dominates at higher temperatures. It may be connected with the anion vacancies, which act as donors, because an excess of cations for the samples with $z>0.06$ is observed.

A sample for $z=0.06$ reveals the SLS EC structure. This structure seems to be accompanied with the impurity conduction and hopping conduction as well as with the formation of ferromagnetic clusters in the $\mathrm{Cr}$ band, which is partly imperfect by cation deficiency (see Table I) from one side and weakly diluted by the $\mathrm{V}^{3+}$ ions forming the $3 d^{2} t_{2 g}^{2} e_{\mathrm{g}}^{0}$ band from the other.

\section{Acknowledgments}

This work is funded from science resources for years 2007-2009 as a research project (project No. N N204 178433)

\section{References}

[1] A.P. Ramirez, R.J. Cava, J. Krajewski, Nature (London) 386, 156 (1997).

[2] K. Ohgushi, T. Ogasawara, Y. Okimoto, S. Miyasaka, Y. Tokura, Phys. Rev. B 72, 155114 (2005).

[3] J. Hemberger, H.-A. Krug von Nidda, V. Tsurkan, A. Loidl, Phys. Rev. Lett. 98, 147203 (2007).

[4] T. Groń, E. Malicka, I. Okońska-Kozłowska, A. Waśkowska, in: Ferrites: Proc. Eight Int. Conf. on Ferrites, The Japan Society of Powder and Powder Metallurgy, Kyoto 2000, p. 211.

[5] T. Groń, A. Krajewski, J. Kusz, E. Malicka, I. Okońska-Kozłowska, A. Waśkowska, Phys. Rev. B 71, 035208 (2005).

[6] S. Kirkpatrick, Phys. Rev. B 16, 4630 (1977).

[7] P.K. Baltzer, H.W. Lehmann, M. Robbins, Phys. Rev. Lett. 15, 493 (1965).

[8] I. Okońska-Kozłowska, J. Krok, Z. Anorg. Allg. Chem. 447, 235 (1978). 